\title{
CAR T Cell Therapy for Hematological Malignancies*
}

\author{
Xin YANG, Gao-xiang WANG, Jian-feng ZHOU \\ Department of Hematology, Tongji Hospital, Tongji Medical College, Huazhong University of Science and Technology, Wuhan \\ 430030, China
}

(C) The Author(s) 2019, corrected publication February 2021

\begin{abstract}
Summary: As a rapidly progressing field in oncology, the adoptive transfer of T cells that have been genetically modified with chimeric antigen receptors (CARs) has shown striking efficacy in the management of hematological malignancies and has been reported in a number of clinical trials. Of note, CAR T cell therapy has shown extraordinary potential, especially in relapsed/refractory patients. However, there are still challenges regarding the further development of this strategy, spanning from engineering and manufacturing issues, to limited applications, to accompanying toxicities. In this review, we will summarize the general knowledge of this novel method, including receptor composition, applications, adverse events and challenges. Additionally, we will propose several comprehensive recommendations.
\end{abstract}

Key words: immune therapy; chimeric antigen receptor T cells; hematological malignancies

Chimeric antigen receptor (CAR) $\mathrm{T}$ cells are those that have been genetically engineered to express recombinant CARs that are capable of recognizing specific antigens on the surface of target cells and then triggering $\mathrm{T}$ cell activation independent of major histocompatibility complex (MHC) molecules. Since CARs provide MHC-independent antigen recognition, some of the mechanisms of immune escape used by tumor cells, such as down-regulation of MHC molecules, can be avoided; in addition, CARs recognize not only proteins but also carbohydrates and lipids ${ }^{[1]}$. While CAR T cell therapy has demonstrated impressive efficacy, there are several serious toxicities that result from this kind of therapy, including cytokine release syndrome (CRS), neurotoxicity, B cell aplasia and graft-versus-host disease (GVHD). Furthermore, failure and relapse with CAR T cell therapy are not uncommon. Further research is needed to minimize adverse events and enhance efficacy.

\section{CAR CONSTRUCTION}

A CAR is a synthetic protein that comprises three main components: an extracellular antigenrecognition domain, a transmembrane domain, and an intracellular signaling domain (fig. 1) ${ }^{[2,3]}$. Firstgeneration CARs contain an single-chain variable

Xin YANG, E-mail: yang_xinnn@163.com

\#Corresponding author, E-mail: zhougene@medmail.com.cn ${ }^{*}$ This project was supported by the Key Program of the National Natural Science Foundation (NNSF) of China (No. 81230052 and No. 81630006). fragment $(\mathrm{ScFv})$ linked to the intracellular signaling domain of $\mathrm{CD} 3 \zeta$, which provides the required signal 1 (the interaction between TCR-CD3 complexes and peptide presented by MHC molecules) to activate $\mathrm{T}$ cells; however, these CARs have no costimulatory molecules and fail to produce enough cytokines, thus leading to limited activation, proliferation and in vivo antitumor effects of $\mathrm{T}_{\text {cells }}{ }^{[4]}$. Second-generation CARs include the addition of costimulatory domains such as CD27, CD28, CD137 (4-1BB), CD134 (OX40), ICOS and CD244, which provides a second activation

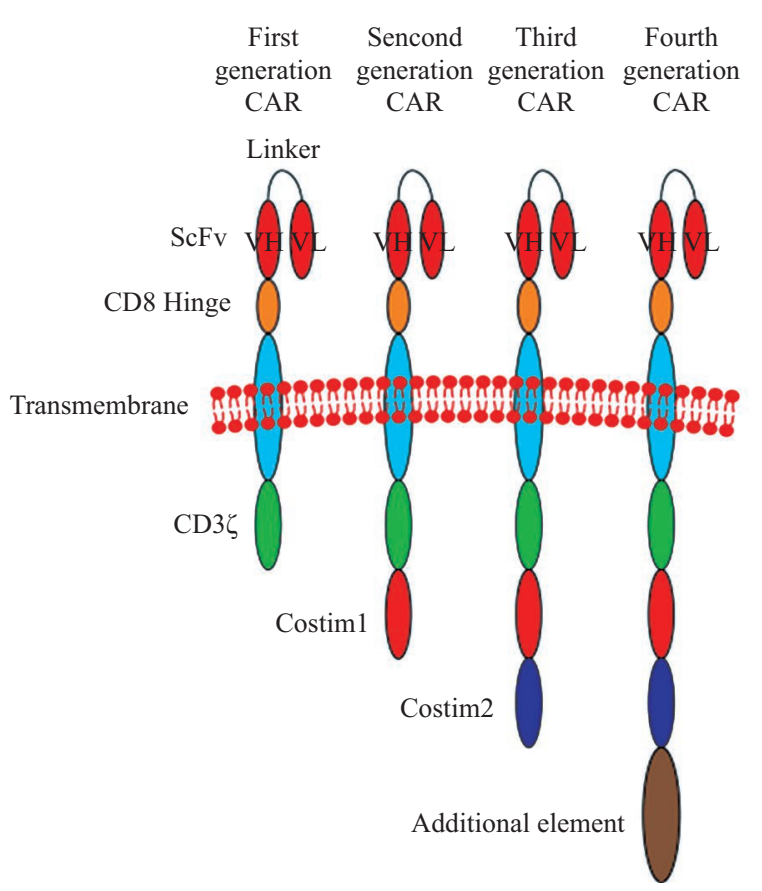

Fig. 1 The general structure of CARs of different generations 
signal enhancing signal 1 . Delivery of signal 1 and signal 2 in the second-generation CARs brings about continuous proliferation of $\mathrm{T}$ cells and secretion of cytokines, leading to substantially more potency in killing target cells in vivo than had been seen with first-generation $\mathrm{CARS}^{[5,6]}$. To enhance the sensitization of CAR $\mathrm{T}$ cells, third-generation CARs include two or more costimulatory molecules that promote improvement of $\mathrm{T}$ cell survival, cytokine production, and antitumor potential ${ }^{[7]}$. In addition to their relatively high efficacy, the third-generation CARs can reduce the undesirable anti-inflammatory effect of IL-10 as a result of the addition of an OX40 signaling domain ${ }^{[8]}$. Nevertheless, the third-generation CARs have a risk of signal leakage and probably produce an excess of cytokines. Unfortunately, improved outcomes were not achieved in clinical trials. Fourth-generation CARs include the combination of second-generation CARs with an additional element, such as the tumorkilling cytokine IL-12. This type of CAR has improved tumor eradication by releasing cytokines into the tumor microenvironment and recruiting immune cells without prior conditioning ${ }^{[9]}$. In addition, it has been used to treat virus infections, metabolic disorders and autoimmune diseases in addition to cancers ${ }^{[10]}$. To date, research on third-generation and fourth-generation CARs has been limited, and they have relatively few applications in the clinic. Moreover, whether the safety and efficacy of third-generation and fourth-generation CARs are better than those of second-generation CARs has not yet been determined. Additionally, the first and second FDA-approved CAR T cells, tisagenlecleucel (Kymriah; Novartis) and axicabtagene ciloleucel (Yescarta; Kite Pharma), respectively, are both secondgeneration CAR T cells. At present, the CAR T cells that have been broadly applied in the clinic have been second-generation.

\section{APPLICATIONS OF CAR T CELLS IN VARIOUS HEMATOLOGICAL MALIGNANCIES}

\subsection{CAR T Cells for Acute Lymphoblastic Leukemia}

Acute lymphoblastic leukemia (ALL) is a malignant hematological disease with abnormal primitive cells and excessive proliferation of naive cells in the bone marrow. CAR T cell therapy is the most suitable for the treatment of ALL, especially fatal $\mathrm{r} / \mathrm{r}$ B-ALL, and this strategy has shown remarkable efficacy. Although it is not the first target to be studied, $\mathrm{CD} 19$, an essential biomarker of the B cell lineage, is the most mature and effective target, showing higher expression in B-ALL, while CD20 and immunoglobulin light chains are also potential targets ${ }^{[11,12]}$. The group at Memorial Sloan Kettering Cancer Center (MSKCC) first reported an adult B-ALL patient treated with anti-CD19 CAR T cells, and the patient maintained remission for 8 weeks before allogeneic hematopoietic stem cell transplantation (allo-HSCT) ${ }^{[13]}$. To date, clinical trials of anti-CD19 CAR T cells for patients with $\mathrm{r} / \mathrm{r}$ B-ALL have shown promising complete remission (CR) and partial remission (PR) rates. The groups from the University of Pennsylvania (UPenn) and Children's Hospital of Philadelphia have published their results of treating $30 \mathrm{r} / \mathrm{r}$ ALL patients with antiCD19 CAR T cells. A total of 27 patients achieved CR (90\%), 22 of whom had an minimal residual disease (MRD)-negative $\mathrm{CR}^{[14]}$. Of note, 2 patients had central nervous system (CNS) blasts prior to infusion of CAR $\mathrm{T}$ cells, and these blasts cleared after infusion with no CNS relapse. Another interesting study reported at the 2016 America Society of Hematology (ASH) annual meeting enrolled 57 pediatric and young adult patients with $\mathrm{r} / \mathrm{r}$ ALL treated by industry-manufactured and successfully transferred CAR T cells. Among those, 29 patients reached day 28 , which was prior to the data cutoff and 83\% (24/29) achieved CR or CRi and were MRD negative. Two patients did not respond ${ }^{[15]}$. Last year, updated results on 75 pediatric and young adult patients with ALL undergoing CD19 CAR T therapy showed an overall response rate (ORR) of $81 \%$ within three months, including a $60 \% \mathrm{CR}$ rate ${ }^{[16]}$.

Although CD19 is an ideal target for CAR T cell therapy in ALL, it is often administered in B cell malignancies but has limited efficacy in $T$ cell malignancies. A study reported findings about CAR T cells engineered to target CD5 and demonstrated that anti-CD5 CAR $T$ cells had the ability to effectively eliminate specific $\mathrm{T}$ cell lines expressing $\mathrm{CD} 5^{[17]}$. CAR $\mathrm{T}$ cells have also been manufactured to target CD4, and these have shown great potency against $\mathrm{T}$ cell lymphoma models in vivo and CD4-positive leukemia cell lines in vitro ${ }^{[18]}$.

Currently, several clinical trials of multitargeted CAR T cell therapy to treat ALL are underway, including tandem anti-CD19-CD22 CAR T cells using a single CAR targeting both antigens and a combination of anti-CD19 CAR T cells and anti-CD20 CAR T cells targeting each antigen individually. A recent clinical trial enrolled 27 patients with $\mathrm{r} / \mathrm{r}$ B-ALL treated with anti-CD19 and anti-CD22 CAR T cells; 24 patients reached $\mathrm{CR}$, including 7 patients who had received prior HSCT, and 13 patients achieved MRD-negative CR, as assessed by flow cytometry. Continuous remission was achieved, with six-month overall survival (OS) rate of $79 \%$ and an event-free survival rate of $72 \%$. The results indicated that multitargeted CAR T cell therapy was feasible and might be a promising approach ${ }^{[19]}$.

In general, these studies provide strong evidence to support the development of CAR T cell therapies for $\mathrm{B}$ cell and T cell ALL.

\subsection{CAR T Cells for Acute Myeloid Leukemia}

Acute myeloid leukemia (AML) is the most 
common type of leukemia and is characterized by monoclonal proliferation of immature myeloid cells in the bone marrow. Unfortunately, AML has not shown the same successful outcomes with CAR T cell therapy as ALL. An early focus of CARs for AML has been on CD123 and CD33 as targets. In 2015, the group from The General Hospital of the People's Liberation Army (PLAGH) first reported the use of CD33 CAR T cell therapy in the treatment of $r / r$ AML. In this patient, the tumor burden was significantly reduced in the bone marrow after 2 weeks of CAR T cell infusion. However, the disease progressed after 9 weeks of infusion ${ }^{[20]}$. CD123 has emerged as a potential new antigen target. Nevertheless, the on-target-off-tumor effects of antiCD123 CAR T cells have limited their potency, as CD123 is also expressed on normal tissues, such as endothelial cells and monocytes, though at lower levels relative to those on AML cells. To translate CD123 CAR T cell therapy to the clinic, further research is needed $^{[21]}$.

Notably, an increasing number of antigens have been pursued as new targets, including Lewis-Y (LeY) antigen and CLEC12A. It has been reported in a phase 1 trial that 4 adults with relapsed AML were treated with anti-LeY CAR T cells. Three of those patients entered the study with MRD; 1 had cytogenetic remission lasting for 5 months, while the others had stable disease (SD) lasting 1.5 to 23 months. Nevertheless, all patients experienced disease progression (DP) despite persistence of CAR T cell ${ }^{[1]}$. Encouragingly, when anti-CLEC12A-CD33 CAR T cells (ICG136) were applied to a 44-year-old female patient with refractory AML, she tolerated this treatment well and reached MRD negativity after 19 days of infusion; she underwent allo-HSCT after 44 days of infusion and has been in $\mathrm{CR}$ ever since ${ }^{[22]}$. It is worth mentioning that in order to increase the viability of CD33 CAR T cell therapy as a potential approach for AML, a CD33 CAR transiently expressing mRNA has been created ${ }^{[23]}$.

In conclusion, these studies have confirmed the effectiveness and potential of CAR T cells in the treatment of $\mathrm{r} / \mathrm{r}$ AML, although several issues remain to be solved.

2.3 CAR T Cells for Chronic Lymphocytic Leukemia

Chronic lymphocytic leukemia (CLL) is a chronic lymphocytic, malignant, clonal proliferative disease that has great heterogeneity in individuals, disease progression, treatment response and clinical prognosis, with allo-HSCT being the only approach that so far results in cure ${ }^{[24]}$. In the last several years, significant progress has been witnessed in terms of CAR T cell therapy for relapsed and high-risk CLL. In 2011, the group from UPenn reported preliminary yet striking clinical results of treatment with anti-CD19 CAR $\mathrm{T}$ cells in 3 patients with advanced CLL, including $2 \mathrm{CRs}^{[25]}$. A clinical trial containing $14 \mathrm{CLL}$ patients reported a 57\% ORR (4 CRs and 4 PRs) after CAR T cell therapy ${ }^{[26]}$, and all patients achieved $\mathrm{CR}$ and MRD negativity without relapse at the 4-year follow-up.

Data from the National Cancer Institute (NCI) regarding the use of anti-CD19-CD28 $\zeta$ CAR T cells to treat CLL are also promising. Three out of 4 patients achieved CRs lasting from 14 to 23 months $^{[27]}$. Studies have shown that CAR T cell antitumor effects correlate with the degree of persistence of CAR $\mathrm{T}$ cells in each individual patient, confirming the importance of pharmacokinetic factors in determining antitumor efficacy $^{[28]}$.

It is worth noting that the Medical College of Wisconsin has conducted a trial of multitargeted CAR T cell therapy targeting both CD19 and CD20, and the outcomes may guide our directions for development of CAR T cell therapy.

In general, the ORR of treatment for CLL with CAR T cells is $53 \%{ }^{[29]}$, but only $26 \%$ of patients can achieve persistent remission ${ }^{[30]}$. On the whole, these data highlight the potency of CAR $\mathrm{T}$ cell therapy in $\mathrm{r} / \mathrm{r}$ CLL, but further study is needed to enhance the effectiveness and stability.

\subsection{CAR T Cells for Non-Hodgkin's Lymphoma}

Non-Hodgkin's lymphoma (NHL) is one of the most common lymphatic malignancies in China. In recent years, despite substantial advances in chemotherapy, radiotherapy and HSCT, the mortality rate of NHL has not been decreased. New therapies are imperative for patients who are resistant to standard strategies, and CAR T cell therapy has recently gained widespread interest owing to its striking success in $r / r$ lymphomas.

CD19, a transmembrane glycoprotein involved in the activation of B cells, is uniformly expressed at all stages of B cell development and is regarded as an ideal target. As expected, anti-CD19 CAR T cells have shown impressive potency in the treatment of chemotherapy-resistant lymphomas, leading to enduring CRs lasting for more than 2 years in some patients with refractory diffuse large B cell lymphoma (DLBCL). Of note, it has been reported that the ORR achieved through this strategy ranged from 59 to $88 \%$, with $50 \%$ of the patients achieving CR in the three reported series of refractory lymphomas ${ }^{[31-33]}$. In a trial involving 108 patients with $\mathrm{r} / \mathrm{r}$ DLBCL, transformed follicular lymphoma (FL) and primary mediastinal large B cell lymphoma (PMBCL) treated with antiCD19 CAR T cells, the ORR and CR rate were $82 \%$ and $54 \%$, respectively ${ }^{[34,35]}$.

CD22 is expressed in B cell progenitors and differentiated B cells, although it is a more mature antigen than CD19. CD22 is also highly and homogeneously expressed in B cell leukemias and lymphomas; therefore, it may be an attractive target for these diseases. A trial of anti-CD22 CAR T cells 
reported that 4/9 patients (some relapsed with CD19negative disease after CD19 CAR T cell therapy) achieved MRD-negative $\mathrm{CRs}^{[36]}$.

CD20 is also a very well-known target for B cell lymphomas, and the anti-CD20 monoclonal antibody rituximab has substantially changed the outcome of NHL patients ${ }^{[37]}$. In an early trial of CD20 CAR T cell treatment for 7 patients with $\mathrm{r} / \mathrm{r}$ NHL, 1 achieved PR, 2 patients had continuous CRs, and 4 patients had $\mathrm{SD}^{[38]}$. Another trial of third-generation anti-CD20 CAR T cells enrolled 4 patients with relapsed mantle cell lymphoma (MCL) and indolent B cell lymphoma. Two patients without evaluable disease were free of progression from 12 to 24 months, and another patient had an objective $\mathrm{PR}^{[7]}$.

In addition, CD23 and orphan tyrosine kinase receptor (ROR1) are also potential targets for B cell lymphomas, and CAR T cell therapy has brought new hope for the treatment of $\mathrm{r} / \mathrm{r}$ NHL.

\subsection{CAR T Cells for Hodgkin's Lymphoma}

Hodgkin's lymphoma (HL) originates from B cells, but HL cells have lost B cell-specific antigens and express CD30 to a high degree. As a result, CD30 is an ideal target for CAR T cell therapy of HL. Although CD30 is also expressed on several activated T cells, making it more challenging to develop CD30 CAR $\mathrm{T}$ cell therapy, there have been several encouraging clinical outcomes. In a phase 1 study on 7 patients with $\mathrm{r} / \mathrm{r}$ HL treated with anti-CD30 CAR $\mathrm{T}$ cells, 1 achieved CR lasting more than 2.5 years after the second infusion of CAR T cells, 1 achieved continued CR for nearly 2 years, and 3 had transient SD. Notably, no toxicities attributable to CD30 CAR T cell therapy were observed ${ }^{[39]}$. Another phase 1 study enrolled 9 patients with r/r, CD30-positive HL and NHL (7 with $\mathrm{HL}$ and 2 with CD30-positive anaplastic large cell lymphoma) treated with anti-CD30 CAR $\mathrm{T}$ cells ${ }^{[39]}$. Seven of these patients who had either relapsed or had DP after treatment with brentuximab vedotin tolerated CAR $T$ cell infusion well and had no accompanying adverse events. Of note, 3 patients achieved CR, and 3 patients remained in SD.

Furthermore, CD33-directed CAR T cells have shown potent anti-lymphoma effects in different tumor models. In a phase 1 study, 18 patients (17 with HL and 1 with cutaneous anaplastic large cell lymphoma) were treated with anti-CD30 CAR T cells ${ }^{[40]}$. Seven patients achieved PR, and 6 reached SD; the treatment was well tolerated and there was an absence of toxicity.

These studies have demonstrated the safety, tolerability, and great potential of CD30 CAR T cell therapy, which may bring significant benefit to patients suffering from $\mathrm{r} / \mathrm{r}$ HL.

\subsection{CAR T Cells for Plasma Cell Disease}

Multiple myeloma (MM) is a malignancy of plasma cells, which are the final developmental stage of
B cells. The lack of an ideal target has limited strategies to manage this disease. For an antigen to be considered an appropriate target for CAR T cell therapy for MM, the specific antigen must be expressed on the surface of myeloma cells but not on the surface of normal cells to avoid severe on-target-off-tumor toxicity from CAR $\mathrm{T}$ cell activation.

CD138, also known as syndecan 1 , is a surface protein highly expressed on both normal and malignant plasma cells and has been considered an attractive target. A Chinese group reported their data about CD138 CAR $\mathrm{T}$ cell therapy for 5 refractory patients, and SD was achieved in 4 out of 5 patients ${ }^{[4]]}$. Nevertheless, CD138 is not specifically expressed by myeloma cells and is also expressed by epithelial cells, raising some issues concerning the specificity and toxicity of this strategy.

Recently, B cell maturation antigen (BCMA), as a surface protein involved in all stages of the differentiation and maturation of $\mathrm{B}$ cells, has drawn increasing attention as an appropriate target for CAR $\mathrm{T}$ cell therapy for MM. Notably, BCMA is also highly expressed on myeloma cells. Preliminary results from the first phase 1 trial of CAR T cell therapy targeting BCMA or CD269 in patients with MM have been published ${ }^{[42]}$. Among 12 patients with advanced disease, 1 patient achieved stringent $\mathrm{CR}$ that was sustained for more than 3 months, whereas another achieved very good partial response (VGPR) lasting for 8 weeks. Although higher doses of the CAR T cells were associated with better responses, they also correlated with increased adverse events such as CRS. Compared to CD138, BCMA is a better candidate because of its absence in most tissues; therefore, CAR T cells targeting BCMA can be effective and have achieved great outcomes in the clinic. Moreover, a recent report of treatment of one case of polyneuropathy, organomegaly, endocrinopathy, monoclonal gammopathy, and skin changes (POEMS) syndrome, which did not respond well to traditional therapies, with anti-BCMA CAR $\mathrm{T}$ cells showed that the patient achieved stringent $\mathrm{CR}$, highlighting the great potential of BCMA CAR T cell therapy in other plasma cell dyscrasias in addition to $\mathrm{MM}^{[43]}$.

Even though normal plasma cells do not have strong expression of CD19, its expression has been observed at low but slightly higher levels than those seen on malignant plasma cells, as previously reported. A successful example of CD19 CAR T cell therapy has been published in which a 43-year-old patient with 9 prior lines of treatment achieved remission via the use of CTL019 cells ${ }^{[44]}$. In this report, the approach was well tolerated, and CRS did not develop; importantly, CTL019 cells were detected in both blood and bone marrow for up to several days after CAR T cell infusion. Moreover, other 9 patients were given the same treatment, with more than half achieving remission ${ }^{[44]}$. An update of this clinical trial featuring a series of 12 
patients was presented at $\mathrm{ASH}^{[45]}$. Ten of the 12 patients enrolled were infused with CTL019, and 6 patients had VGPR, 2 had PR, and 2 had progressive disease (PD). Of note, only minor adverse events were observed.

Overall, CAR T cell therapy is a promising strategy in MM.

\section{ADVERSE EVENTS OF CAR T CELLTHERAPY}

\subsection{Cytokine Release Syndrome}

CRS, which is a non-antigen-specific systemic inflammatory response as a result of generalized immune activation associated with CAR $\mathrm{T}$ cell expansion and marked elevations in cytokines, is one of the most common adverse events in CAR $\mathrm{T}$ cell therapy. Although accurate predictors of CRS have not been defined in clinical practice due to technical difficulties in monitoring serum cytokines, there is accumulating evidence that the incidence and severity of CRS seem to correlate with tumor burden, use of lymphodepleting therapy, preexisting endothelial activation, infusion dose of CAR T cells and increased levels of CAR T cell proliferation/kinetics ${ }^{[26,46-49]}$.

CRS manifests as a series of symptoms, including fever, fatigue, myalgias, malaise, nausea, anorexia, hypotension, hypoxia, coagulopathy, and capillary leak, and is often accompanied by organ damage (renal impairment, cardiac dysfunction, hepatic failure, etc. $)^{[50]}$. Of note, CRS has been reported to occur in nearly $30 \%-94 \%$ of patients from 1 to 22 days after CAR T cell infusion, and the median time of onset is 2-3 days ${ }^{[31,51]}$. The first clinical sign of CRS to be observed in most patients is high fever, which can has risen to more than $40^{\circ} \mathrm{C}$ in several individuals ${ }^{[52]}$.

A published rating scale for CRS combines laboratory findings with clinical features. The criteria for severe CRS, which is defined as CRS requiring pharmacologic and medical intervention, are fevers higher than $38^{\circ} \mathrm{C}$ lasting for at least 3 consecutive days and elevation of at least one type of serum cytokine by at least 250-fold or two types of serum cytokines by 75fold, and one clinical sign of severe toxicity ${ }^{[48]}$. Another system of grading has been reported, in which grade 1 CRS requires only supportive management; grade 2 CRS criteria include requirement of intravenous injection (IV) therapies, grade 2 creatinine elevation, grade 3 transaminitis, neutropenic fevers, and any other indications for hospitalization; grade $3 \mathrm{CRS}$ includes grade 3 creatinine elevation, grade 4 transaminitis, hypotension responding to IV fluids or low-dose vasopressors, hypoxia requiring supplemental oxygen, and coagulopathy needing fresh frozen plasma or cryoprecipitate; and grade 4 CRS includes lifethreatening complications, such as hypoxia requiring ventilator support or hypotension demanding highdose vasopressors ${ }^{[26]}$.
In the clinic, severe sepsis, as a common complication of CAR T cell therapy, is difficult to differentiate from CRS. Both are characterized by fever and elevation of serum cytokines. Laboratory examinations, such as microbiologic culture and specific nucleic acid and antibody assays, may contribute to the diagnosis of microbial infection. Furthermore, IFN- $\gamma$ is rarely significantly elevated in most severe sepsis cases, although IL-6 and IL-10 are very high, which is quite different from CRS resulting from CAR T cell infusion and might be helpful for differentiation $^{[53]}$.

Current management strategies include supportive care (antipyresis, intravenous fluids, blood component transfusions, vasopressors, etc.) and tocilizumab, a monoclonal antibody against both membrane-bound and soluble IL-6 receptor, and/or steroids for higher grades of this syndrome. Tocilizumab and steroids are able to control a majority of CRS cases. Nonetheless, resistant CRS, characterized by no clinical improvement at any time even with the use of tocilizumab and steroids ${ }^{[54]}$, may occur in a minority of patients, and in these patients, the mortality is very high ${ }^{[29]}$.

\subsection{Neurotoxicity}

Neurotoxicity is another common side effect correlated with CAR T cell therapy that occurs in patients even without detectable CNS blasts before infusion of CAR T cells. Neurologic symptoms vary widely, ranging from mild headache, confusion, visual hallucination, delirium, or tremors to acute encephalopathy, seizures, and rarely cerebral edema ${ }^{[46,52,55,56]}$. The onset of this unique toxicity is less predictable than that of CRS and occurs 4-5 days after CAR T cell infusion, usually after the onset of CRS, but neurotoxicity does not exactly follow the same time course as systemic CRS. The pathogenesis is unclear, which may be associated with the diffusion of cytokines into the brain or trafficking of $\mathrm{T}$ cells to the brain ${ }^{[49,57]}$. Despite several devastating cases requiring immediate attention, most patients do not develop radiographic or clinical signs of cerebral edema, and neurotoxicity is usually self-limiting within days; the majority of cases can be reversed, especially with appropriate treatment ${ }^{[49]}$. Current management for this unique neurotoxicity is aimed at reducing the inflammatory response. Siltuximab, a direct IL-6 antagonist, may play an important role since it is able to prevent continuous IL-6 translocation across the blood-brain barrier $(\mathrm{BBB})^{[46]}$. Moreover, high-dose systemic corticosteroids are indicated and have good CNS penetration in severe conditions ${ }^{[50]}$. Appliance of levetiracetam or other antiepileptic agents can also be considered in the setting of severe neurologic dysfunction as prophylaxis for seizures ${ }^{[58]}$. Additional studies are needed to determine the mechanism, risk factors, and optimal management of neurotoxicity after CAR T cell therapy. 


\subsection{Hemophagocytic Lymphohistiocytosis/Macrophage Activation Syndrome}

Hemophagocytic lymphohistiocytosis (HLH) is a rare condition that presents with fever, splenomegaly, hyperferritinemia, coagulopathy, hypertriglyceridemia, and cytopenia due to inappropriate immune activation and cytokine release ${ }^{[59]}$, and HLH even occurs in patients with low-grade CRS. In particular, severe CRS can evolve into HLH; therefore, clinicians must be aware of this condition to avoid fatal outcomes. A definition for HLH/macrophage activation syndrome (MAS) after CAR T cell therapy has been proposed in relation to the CAR $\mathrm{T}$ cell therapy-associated toxicities (CARTOX) score, which includes a peak serum ferritin level of $>10000 \mathrm{ng} / \mathrm{mL}$ and any two of the following findings: grade $\geq 3$ oliguria or elevation in serum creatinine levels; grade $\geq 3$ pulmonary edema; grade $\geq 3$ elevation in serum bilirubin, alanine aminotransferase, or aspartate aminotransferase levels; and presence of hemophagocytosis in the bone marrow $^{[52]}$. Current strategies for these patients should include anti-IL-6 therapy, similar to what is done for CRS, and corticosteroids if clinically necessary, and this strategy is able to reverse this clinical condition. If the situation is not improved within $48 \mathrm{~h}$, additional therapy, including etoposide and intrathecal cytarabine, should be taken into consideration, especially for neurotoxicity-associated $\mathrm{HLH}^{[50]}$. Additional studies are critical to understand the pathophysiology and patterns of this adverse event in order to effectively manage these patients and improve their outcomes.

\subsection{B Cell Aplasia}

CAR T cell therapies targeting B cells, such antiCD19 CAR T cells, can lead to absolute and functional hypogammaglobulinemia because they are not able to discriminate malignant from normal B cells ${ }^{[60]}$. Ontarget-off-tumor effects, such as B cell aplasia induced by CD19-targeted CAR T cells, are expected because CAR T cells attack normal tissues that share the same expression with the targeted cells. In the ELIANA trial, the incidence of B cell aplasia at 6th month was $83 \%$, and B cell aplasia was present in all patients responding to CAR T cell therapy ${ }^{[50]}$. On the other hand, occurrence of long-lasting CR along with B cell aplasia demonstrates the functional persistence of CAR T cells in vivo and highlights the potential for a paradigm shift in adoptive immunotherapy ${ }^{[26]}$. Immunoglobulin replacement therapy remains the most effective strategy to manage B cell aplasia and hypogammaglobulinemia but needs to be individualized according to the disease, therapy and serum antibody titers ${ }^{[61]}$.

\subsection{GVHD}

In several cases, patients receive infusion of specific CAR-transduced allogeneic $T$ cells from donors, and these patients have a risk of GVHD. In a report, GVHD resulted from donor-derived allogeneic anti-CD19 CAR T cell infusion occurred 3-4 weeks after treatment in 2 patients. One case manifested as aggravated hyperbilirubinemia and elevated aminotransferases, and the other appeared as chronically aggravated skin damage. Fortunately, both cases were easily controlled with short-term use of corticosteroids $^{[62]}$.

\section{RESISTANCE AND RELAPSE WITH CAR T CELL THERAPY}

Although extraordinary responses have been produced by CAR $\mathrm{T}$ cell therapy in many patients, there are still a number of cases that are resistant to this strategy. There may be several factors, including the phenotype of the infused CAR T cells as well as tumor-derived factors, that affect the efficacy of CAR $\mathrm{T}$ cells and result in their failure to be activated and proliferate in vivo upon tumor antigen encounter ${ }^{[63]}$. Current approaches to reduce the impact of factors related to the failure of CAR T cell therapy include the concomitant use of anti-PD-1/PD-L1 antibodies and optimization of CAR T cells, empowering which to secrete cytokines capable of inhibiting tumor-related suppressive molecules ${ }^{[64-66]}$.

Despite CAR T cell expansion and achievement of $\mathrm{CR}$, relapse commonly occurs, and the target antigen may remain on the surface of the tumor cells or not ${ }^{[67,68]}$. Target-positive relapses occur in patients with loss of functioning CAR T cells due to the host immunological processes to reject the CAR T cells. Similarly, targetnegative relapses are not uncommon. CD19-negative relapse occurs in $14 \%$ of all adult B cell ALL patients according to investigation at the MSKCC ${ }^{[69]}$. There may be several mechanisms playing a role in antigen escape, including diminishment or disappearance of the antigen that is recognized by the antigen-binding domain of CAR $\mathrm{T}$ cells ${ }^{[68]}$. In a recent study, it has been suggested that CARs trigger reversible antigen loss through trogocytosis, an active process in which the target antigen is transferred to $\mathrm{T}$ cells, thereby decreasing the density of targeted antigen on tumor cells and decreasing $\mathrm{T}$ cell activity by promoting CAR $\mathrm{T}$ cell fratricide ${ }^{[70]}$. In addition, transcription factor defects and transformation of leukemic cells (lymphoid to myeloid) contribute to relapses in a minority of cases $^{[71]}$.

Novel strategies to prevent antigen loss and improve outcomes include targeting multiple molecules besides CD19, such as CD20, CD22 and CD123 [36, 72], and allo-HSCT, which may reduce the incidence of CD19-negative relapses after $\mathrm{CR}^{[73]}$. Additionally, bispecific CAR T cells are currently being developed and have shown promise in overcoming resistance or relapse due to antigen $\operatorname{loss}^{[74]}$.

To expand the flexibility and efficacy of CAR 
$\mathrm{T}$ cell therapy, a split, universal, and programmable (SUPRA) CAR system has been proposed that has the ability to switch targets without re-engineering $\mathrm{T}$ cells, enhance the activation of CAR $\mathrm{T}$ cells and respond to multiple antigens. In other words, the SUPRA CAR is a two-section receptor system containing a universal receptor and a separate $\mathrm{ScFv}$ targeting specific antigens ${ }^{[75]}$. Similarly, universal $\mathrm{T}$ cells have been designed by disrupting the TCR gene and/or human leukocyte antigen (HLA) class I molecules of allogeneic $\mathrm{T}$ cells. Thus, the expression of the TCR or HLA class I antigen is eliminated, and the CAR T cells can avoid being recognized, leading to reduced occurrence of $\mathrm{GVHD}^{[76]}$.

\section{CONCLUSION}

With the rapid developments in cellular immunity as well as transplantation immunology, the relationship between CAR T cell therapy and allo-HSCT has been widely discussed. The outcomes after CAR $\mathrm{T}$ cell therapy are variable depending on the disease, the patients, and the construction of CARs, all of which need to be taken into consideration when determining the relative roles of CAR T cell therapy and allo-HSCT. However, there have not been carefully designed clinical trials that can draw definite conclusions yet. Based on our clinical experience, we make several comprehensive recommendations: (1) For patients with refractory malignancies, CAR $\mathrm{T}$ cell therapy may be used before allo-HSCT to reduce the tumor burden or even induce remission and could increase the possibility of successful allo-HSCT; (2) For patients with relapsed B cell malignancies after allo-HSCT, especially for B-ALL, CAR T cell therapy may be more effective than other strategies, such as donor lymphocyte infusion; (3) For patients with refractory malignancies, CAR T cell therapy may achieve continuous remission, especially in NHL and CLL, and could even replace allo-HSCT; (4) For patients who initially achieve but then lose B cell aplasia after CAR T cell infusion, subsequent alloHSCT should be considered; (5) For patients whose liquid biopsy or other tumor-related factors indicate progression of disease, a timely allo-HSCT should be performed. These recommendations are empirical, and further study is urgent. CAR T cell therapy has brought new hope to the treatment of malignant hematological diseases such as ALL, CLL, AML, NHL, HL and MM. However, there are still problems to be solved, including side effects such as CRS, on-target-offtumor effects and neurotoxicity, optimal cell dosage, enhancement of specificity and efficacy, resistance to this strategy, relapses after a transient period of CR, and treatment before or after CAR T cell therapy. Given the complexity and novelty of this therapy, the issues to be solved are significantly challenging. Fortunately, we have witnessed great potential of this strategy from preclinical investigations and clinical trials, and we believe that the development of CAR T cell therapy and other combined strategies can provide substantial clinical benefits to patients.

\section{Conflict of Interest Statement}

The authors have declared that they have no conflicts of interest.

\section{REFERENCES}

1 Ritchie DS, Neeson PJ, Khot A, et al. Persistence and efficacy of second generation CAR $\mathrm{T}$ cell against the LeY antigen in acute myeloid leukemia. Mol Ther, 2013,21(11):2122-2129

2 Jackson HJ, Rafiq S, Brentjens RJ. Driving CAR T-cells forward. Nat Rev Clin Oncol, 2016,13:370-383

3 Fesnak AD, June CH, Levine BL. Engineered T cells: the promise and challenges of cancer immunotherapy. Nat Rev Cancer, 2016,16:566-581

4 Heuser C, Hombach A, Losch C, et al. T-cell activation by recombinant immunoreceptors: impact of the intracellular signalling domain on the stability of receptor expression and antigen-specific activation of grafted T cells. Gene Ther, 2003,10(17):1408-1419

5 Song DG, Ye Q, Poussin M, et al. CD27 costimulation augments the survival and antitumor activity of redirected human T cells in vivo. Blood, 2012,119(3):696-706

6 Milone MC, Fish JD, Carpenito C, et al. Chimeric receptors containing $\mathrm{CD} 137$ signal transduction domains mediate enhanced survival of $\mathrm{T}$ cells and increased antileukemic efficacy in vivo. Mol Ther, 2009,17(8):1453-1464

7 Till BG, Jensen MC, Wang J, et al. CD20-specific adoptive immunotherapy for lymphoma using a chimeric antigen receptor with both CD28 and 4-1BB domains: pilot clinical trial results. Blood, 2012,119(17):39403950

8 Hombach AA, Heiders J, Foppe M, et al. OX40 costimulation by a chimeric antigen receptor abrogates CD28 and IL-2 induced IL-10 secretion by redirected CD4(+) T cells. Oncoimmunology, 2012,1(4):458-466

9 Pegram HJ, Lee JC, Hayman EG, et al. Tumor-targeted T-cells modified to secrete IL-12 eradicate systemic tumors without need for prior conditioning. Blood, 2012,119(18):4133-4141

10 Chmielewski M, Abken H. TRUCKs: the fourth generation of CARs. Expert Opin Biol Ther, 2015,15(8): $1145-1154$

11 Gill S, Maus MV, Porter DL. Chimeric antigen receptor T cell therapy: 25 years in the making. Blood Rev, 2016, 30:157-167

12 Jain N, O'Brien S. Targeted therapies for CLL: practical issues with the changing treatment paradigm. Blood Rev, 2016,30:233-244

13 Fujiwara H. Adoptive immunotherapy for hematological malignancies using $\mathrm{T}$ cells gene-modified to express tumor antigen-specific receptors. Pharmaceuticals (Basel), 2014,7(12):1049-1068

14 Maude SL, Frey N, Shaw PA, et al. Chimeric antigen receptor T cells for sustained remissio ns in leukemia. $\mathrm{N}$ 
Engl J Med, 2014,371(16):1507-1517

15 Grupp SA, Laetsch TW, Buechner J, et al. Analysis of a global registration trial of the efficacy and safety of CTL019 in pediatric and young adults with relapsed/ refractory acute lymphoblastic leukemia (ALL). Blood, 2016,128:221-221

16 Maude SL, Laetsch TW, Buechner J, et al. Tisagenlecleucel in children and young adults with B-cell lymphoblastic leukemia. N Engl J Med, 2018,378(5):439-448

17 Mamonkin $\mathrm{M}$, Rouce $\mathrm{RH}$, Tashiro $\mathrm{H}$, et al. A T-cell directed chimeric antigen receptor for the selective treatment of T-cell malignancies. Blood, 2015,126(8):983-992

18 Pinz K, Liu H, Golightly M, et al. Preclinical targeting of human T-cell malignancies using CD4-specific chimeric antigen receptor (CAR)-engineered $\mathrm{T}$ cells. Leukemia, 2016,30(3):701-707

19 Huang L, Wang N, Cao Y. CAR22/19 Cocktail Therapy for Patients with Refractory/Relapsed B-Cell Malignancies. Blood, 2018,132:(abstract1408)

20 Wang QS, Wang Y, Lv HY, et al. Treatment of CD33directed chimeric antigen receptor-modified $\mathrm{T}$ cells in one patient with relapsed and refractory acute myeloid leukemia. Mol Ther, 2005,23(1):184-191

21 Tettamanti S, Biondi A, Biagi E, et al. CD123 AML targeting by chimeric antigen receptors: A novel magic bullet for AML therapeutics?. Oncoimmunology, 2014,3:e28835

22 Morsink LM, Walter RB, Ossenkoppele GJ. Prognostic and therapeutic role of CLEC12A in acute myeloid leukemia. Blood Rev, 2019,34:26-33

23 Kenderian SS, Ruella M, Shestova O, et al. CD33specific chimeric antigen receptor $\mathrm{T}$ cells exhibit potent preclinical activity against human acute myeloid leukemia. Leukemia, 2015,29(8):1637-1647

24 Mewawalla P, Nathan S. Role of allogeneic transplantation in patients with chronic lymphocytic leukemia in the era of novel therapies: a review. Ther Adv Hematol, 2014,5(5):139-152

25 Porter DL, Levine BL, Kalos M, et al. Chimeric antigen receptor modified $\mathrm{T}$ cells in chronic lymphoid leukemia. N Engl J Med, 2011,365(8):725-733

26 Porter DL, Hwang WT, Frey NV, et al. Chimeric antigen receptor $\mathrm{T}$ cells persist and induce sustained remissions in relapsed refractory chronic lymphocytic leukemia. Sci Transl Med, 2015,7(303):303ra139

27 Kochenderfer JN, Dudley ME, Kassim SH, et al. Chemotherapy-refractory diffuse large B-cell lymphoma and indolent B-cell malignancies can be effectively treated with autologous T cells expressing an anti-CD19 chimeric antigen receptor. J Clin Oncol, 2015,33(6): 540-549

28 Norelli M, Casucci M, Bonini C, et al. Clinical pharmacology of CAR-T cells: Linking cellular pharmacodynamics to pharmacokinetics and antitumor effects. Biochim Biophys Acta, 2016,1865(1):90-100

29 Mueller KT, Maude SL, Porter DL, et al. Cellular kinetics of CTL019 in relapsed/refractory B-cell acute lymphoblastic leukemia and chronic lymphocytic leukemia. Blood, 2017,130(21):2317-2325

30 Fraietta JA, Lacey SF, Orlando EJ, et al. Determinants of response and resistance to CD19 chimeric antigen receptor (CAR) T cell therapy of chronic lymphocytic leukemia. Nat Med, 2018,24(5):563-571

31 Neelapu SS, Locke FL, Bartlett NL, et al. Axicabtagene ciloleucel CAR T-cell therapy in refractory large B-cell lymphoma. N Engl J Med, 2017(26),377:2531-2544

32 Abramson JS, Chen YB. More on antiCD19 CAR T cells in CNS diffuse large-B-cell lymphoma. N Engl J Med, 2017,377(21):2102

33 Schuster SJ, Svoboda J, Chong EA, et al. Chimeric antigen receptor $\mathrm{T}$ cells in refractory B-cell lymphomas. N Engl J Med, 2017,377(26):2545-2554

34 Neelapu SS. An interim analysis of the ZUMA-1 study of KTE-C19 in refractory, aggressive non-Hodgkin lymphoma. Clin Adv Hematol Oncol, 2017,15(2):117120

35 Locke FL, Neelapu SS, Bartlett NL, et al. Phase 1 results of ZUMA-1: a multicenter study of KTE-C19 anti-CD19 CAR T cell therapy in refractory aggressive lymphoma. Mol Ther, 2017,25(1):285-295

36 Fry TJ, Stetler-Stevenson M, Shah NN, et al. Clinical activity and persistence of anti-CD22 chimeric antigen receptor in children and young adults with relapsed/ refractory acute lymphoblastic leukemia (ALL). Blood, 2015,126(23):(abstract1324)

37 Teo EC, Chew Y, Phipps C. A review of monoclonal antibody therapies in lymphoma. Crit Rev Oncol Hematol, 2016,97:72-84

38 Till BG, Jensen MC, Wang J, et al. Adoptive immunotherapy for indolent non-Hodgkin lymphoma and mantle cell lymphoma using genetically modified autologous CD20-specific T cells. Blood, 2008,112(6):2261-2271

39 Ramos CA, Ballard B, Zhang $\mathrm{H}$, et al. Clinical and immunological responses after CD30-specific chimeric antigen receptor-redirected lymphocytes. J Clin Invest, 2017,127(9):3462-3471

40 Wang CM, Wu ZQ, Wang Y, et al. Autologous T cells expressing CD30 chimeric antigen receptors for relapsed or refractory hodgkin lymphoma: An open-label phase I trial. Clin Cancer Res, 2017,23(5):1156-1166

41 Heffner LT, Jagannath S, Zimmerman TM, et al. BT062, an antibody-drug conjugate directed against CD138, given weekly for 3 weeks in each 4 week cycle: safety and further evidence of clinical activity. Blood, 2015,120(21):4042

42 Ali SA, Shi V, Maric I, et al. T cells expressing an antiB-cell maturation-antigen chimeric antigen receptor causes remission of multiple myeloma. Blood, 2016, 128(13):1688-1700

$43 \mathrm{Xu}$ J, Wang Q, Xu H, et al. Anti-BCMA CAR-T cells for treatment of plasma cell dyscrasia: case report on POEMS syndrome and multiple myeloma. J Hematol Oncol, 2018,11(1):128

44 Garfall AL, Maus MV, Hwang WT, et al. Chimeric antigen receptor $\mathrm{T}$ cells against CD19 for multiple myeloma. N Engl J Med, 2015,373(11):1040-1047

45 Garfall AL, Stadtmauer EA, Maus MV, et al. Pilot study of anti-CD19 chimeric antigen receptor T cells (CTL019) in conjunction with salvage autologous stem cell transplantation for advanced multiple myeloma. Blood, 2016,128(22):(abstract974) 
46 Gust J, Hay KA, Hanafi LA, et al. Endothelial activation and blood-brain barrier disruption in neurotoxicity after adoptive immunotherapy with CD19 CAR-T cells. Cancer Discov, 2017,7(12):1404-1419

47 Brentjens R, Yeh R, Bernal Y, et al. Treatment of chronic lymphocytic leukemia with genetically targeted autologous $\mathrm{T}$ cells: case report of an unforeseen adverse event in a phase I clinical trial. Mol Ther, 2010,18(4):666-668

48 Davila ML, Riviere I, Wang X, et al. Efficacy and toxicity management of $19-28 \mathrm{z}$ CAR $\mathrm{T}$ cell therapy in B cell acute lymphoblastic leukemia. Sci Transl Med, 2014,6(224):224ra25

49 Lee DW, Kochenderfer JN, Stetler-Stevenson M, et al. $\mathrm{T}$ cells expressing CD19 chimeric antigen receptors for acute lymphoblastic leukaemia in children and young adults: a phase 1 dose-escalation trial. Lancet, 2015,385(9967):517-528

50 Neelapu SS, Tummala S, Kebriaei P, et al. Chimeric antigen receptor T-cell therapy - assessment and management of toxicities. Nat Rev Clin Oncol, 2018,15(1):47-62

51 Buechner J, Grupp SA, Maude SL, et al. Global registration trial of efficacy and safety of CTL019 in pediatric and young adult patients with relapsed/ refractory $(\mathrm{R} / \mathrm{R})$ acute lymphoblastic leukemia (ALL): update to the interim analysis. Haematological, 2017,102:178:(abstract S476)

52 Mei H, Jiang $\mathrm{H}, \mathrm{Wu} \mathrm{Y}$, et al. Neurological toxicities and coagulation disorders in the cytokine release syndrome during CAR-T therapy. Br J Haematol, 2017, 181(5):689692

$53 \mathrm{Xu} \mathrm{XJ}$, Tang YM, Liao C, et al. Inflammatory cytokine measurement quickly discriminates gram-negative from gram-positive bacteremia in pediatric hematology/ oncology patients with septic shock. Intensive Care Med, 2013,39(2):319-326

54 Ishii K, Shalabi H, Yates B, et al. Tocilizumab refractory cytokine release syndrome (CRS) triggered by chimeric antigen receptor (CAR)-transduced T cells may have distinct cytokine profiles compared to typical CRS. Blood, 2016,128(22):3358

55 Prudent V, Breitbart WS. Chimeric antigen receptor T-cell neuropsychiatric toxicity in acute lymphoblastic leukemia. Palliat Support Care, 2017,15(4):499-503

56 Wang Z, Guo Y, Han W. Current status and perspectives of chimeric antigen receptor modified $\mathrm{T}$ cells for cancer treatment. Protein Cell, 2017,8(12):896-925

$57 \mathrm{Hu} \mathrm{Y,} \mathrm{Sun} \mathrm{J,} \mathrm{Wu} \mathrm{Z,} \mathrm{et} \mathrm{al.} \mathrm{Predominant} \mathrm{cerebral} \mathrm{cytokine}$ release syndrome in CD19-directed chimeric antigen receptor-modified $\mathrm{T}$ cell therapy. J Hematol Oncol, 2016,9(1):70

58 Pehlivan KC, Duncan BB, Lee DW. CAR-T Cell Therapy for Acute Lymphoblastic Leukemia: Transforming the Treatment of Relapsed and Refractory Disease. Curr Hematol Malig Rep, 2018,13(5):396-406

59 Risma K, Jordan MB. Hemophagocytic lymphohistiocytosis: updates and evolving concepts. Curr Opin Pediatr, 2012,24(1):9-15

60 Bonifant CL, Jackson HJ, Brentjens RJ, et al. Toxicity and management in CAR T-cell therapy. Mol Ther Oncolytics, 2016,3:16011
61 Vairy S, Garcia JL, Teira PB, et al. CTL019 (tisagenlecleucel): CAR-T therapy for relapsed and refractory B-cell acute lymphoblastic leukemia. Drug Des Devel Ther, 2018,12:3885-3898

62 Dai $\mathrm{H}$, Zhang $\mathrm{W}$, Li X, et al. Tolerance and efficacy of autologous or donor-derived $\mathrm{T}$ cells expressing CD19 chimeric antigen receptors in adult B-ALL with extramedullary leukemia. Oncoimmunology, 2015,4(11):e1027469

63 Turtle CJ, Riddell SR, Maloney DG. CD19Targeted chimeric antigen receptor-modified T-cell immunotherapy for B-cell malignancies. Clin Pharmacol Ther, 2016,100(3):252-258

64 Ninomiya S, Narala N, Huye L, et al. Tumor indoleamine 2,3-dioxygenase (IDO) inhibits CD19-CAR T cells and is downregulated by lymphodepleting drugs. Blood, 2015,125(25):3905-3916

65 Pegram HJ, Purdon TJ, van Leeuwen DG, et al. IL12-secreting CD19-targeted cord blood-derived T cells for the immunotherapy of B-cell acute lymphoblastic leukemia. Leukemia, 2015,29(2):415-422

66 Curran KJ, Seinstra BA, Nikhamin Y, et al. Enhancing antitumor efficacy of chimeric antigen receptor $\mathrm{T}$ cells through constitutive CD40L expression. Mol Ther, 2015,23(4):769-778

67 Gardner R, Wu D, Cherian S, et al. Acquisition of a CD19 negative myeloid phenotype allows immune escape of MLL-rearranged B-ALL from CD19 CAR-T cell therapy. Blood, 2016,127(20):2406-2410

68 Sotillo E, Barrett DM, Black KL, et al. Convergence of acquired mutations and alternative splicing of CD19 enables resistance to CART-19 immunotherapy. Cancer Discov, 2015,5(12):1282-1295

69 Park J, Riviere I, Wang X, et al. Impact of disease burden on longterm outcome of 19-28z CAR modified $\mathrm{T}$ cells in adult patients with relapsed B-ALL. J Clin Oncol, 2016,34:(abstract7003)

70 Hamieh M, Dobrin A, Cabriolu A, et al. CAR T cell trogocytosis and cooperative killing regulate tumour antigen escape. Nature, 2019,568(7750):112-116

71 Wei G, Ding L, Wang J, et al. Advances of CD19directed chimeric antigen receptor-modified $T$ cells in refractory/relapsed acute lymphoblastic leukemia. Exp Hematol Oncol, 2017,6:10

72 Haso W, Lee DW, Shah NN, et al. Anti-CD22-chimeric antigen receptors targeting B-cell precursor acute lymphoblastic leukemia. Blood, 2013,121(7):11651174

73 Ruella M, Maus MV. Catch me if you can: leukemia escape after CD19-directed T cell immunotherapies. Comput Struct Biotechnol J, 2016,14:357-362

74 Dufort F, Su L, Wu L, et al. Activity of CAR19 $\mathrm{T}$ cells secreting impact fusion proteins against hematopoietic and solid tumors. Am Soc Clin Oncol, 2018,36(15):e15046

75 Cho JH, Collins JJ, Wong WW. Universal Chimeric Antigen Receptors for Multiplexed and Logical Control of T Cell Responses. Cell, 2018,173(6):1426-1438

76 Zhao J, Lin Q, Song Y. Universal CARs, universal T cells, and universal CAR T cells. J Hematol Oncol, 2018,11(1):132

(Received Apr. 25, 2019; revised Oct. 25, 2019) 